\title{
LOCAL-GLOBAL OPTICAL FLOW FOR IMAGE REGISTRATION
}

\author{
Ammar Zayouna \\ Richard Comley \\ Middlesex University \\ School of Engineering and Information Sciences \\ Middlesex University, London NW4 4BT, UK \\ A.Zayouna@mdx.ac.uk_R.Comley@mdx.ac.uk_D.Shi@mdx.ac.uk \\ Daming Shi
}

\begin{abstract}
Registration is a fundamental task in image processing used to match two or more images taken, for example, at different times, from different sensors, or from different viewpoints. Optical flow is a technique in computer vision area to compute the displacement field of the contents within an image sequence. In the sense of correspondence, image registration and optical flow have very close relation. On the one hand, optical flow is used to do image registration; on the other hand, it is also used to evaluate the performance of image registration. In literature, either local optical flow or global optical flow is studied for image registration. In this paper, an improved optical flow technique, namely Local-Global, which combines the advantages of both techniques, is applied for image registration. Experiments are conducted to demonstrate the effectiveness of this method
\end{abstract}

Keywords: Image registration; global-local optical flow; correspondence estimation; tracking.

\section{INTRODUCTION}

As a fundamental task in image processing, image registration is defined as the process of aligning two or more images so that the shape, size, and spatial relationships of corresponding image contents can be easily matched or related. This concept is shown in Figure 1. The alignment process can be described with a geometrical transformation, namely a spatial mapping:

$$
\mathbf{T}: f_{B} \mapsto f_{A} \Leftrightarrow \mathbf{T}\left(f_{B}\right)=f_{A}
$$

where $\mathbf{T}$ stands for the transformation, $f_{A}$ is the target image, which acts as the reference and $f_{B}$ is the source image, which is to be transformed toward to the reference.

The transformation can be found either by estimating the displacement of each pixel in the image or by finding certain number of parameters that describe the deformation pattern. The former is called nonparametric registration, while the latter is known as parametric registration.

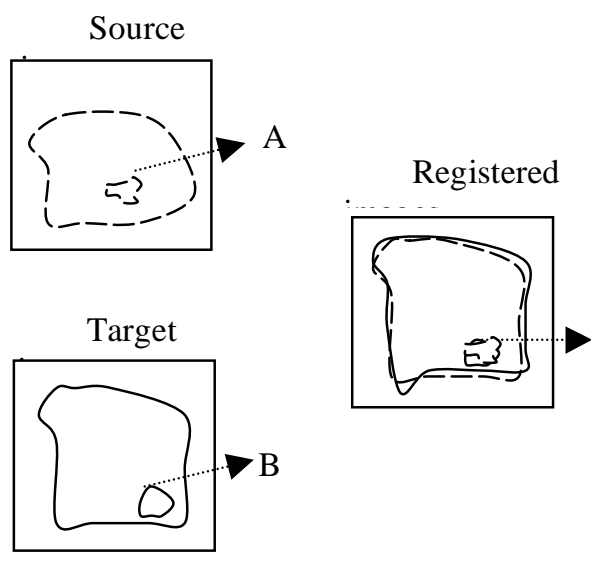

Figure 1: Image registration: the correspondence between point $A$ and point $B$ is found after registration.

Widely used in computer vision, optical flow is a technique to study the motion of contents within an image sequence. In literature there are two comprehensive papers on optical flow algorithms and their performance. (Barron et al., 1994) compared nine classic flow algorithms on the basis of accuracy and density. They classified these algorithms into four groups: differential techniques, energy based techniques, phase based techniques and region matching based techniques. (Liu et al., 1998) extended Barron's work by providing a coordinate system that compares accuracy with 
efficiency. They classified optical flow algorithms into two groups: those that perform a gradient search on extracted structure of the image sequence and those that do not.

In optical flow, the motion is typically represented with velocity vectors originating from each pixel position. These vectors specify how the image pixels move between adjacent images. In this sense of correspondence, the procedure of determining optical flow is essential the nonparametric image registration. (Periaswamy and Farid, 2003) used optical flow in their general framework for medical image registration and achieved very good results. On the other hand, optical flow is also used to evaluate the result of medical image registration (Cooper and Ritter, 2003). A detailed description of the relation between image registration and optical flow can be found in (Lefébure and Cohen, 2001).

The most important assumption for optical flow is that when an image pattern moves, the brightness of a particular point in this pattern keeps constant. With this assumption, the famous optical flow equation can be derived either by chain rule differentiation or by first order Taylor approximation. For a 2D image, the problem is that the solution, namely the velocities in $x$ and $y$ directions (2 unknowns) cannot be uniquely determined with just one single equation. Extra constraints are needed. According to the types of constraints, differential techniques can be classified into local optical flow and global optical flow. Both of these two methods have their advantages and shortcomings. (Bruhn A and Weickert, 2005) proposed a method to combining the local optical flow to the global one.

However, when considered with image registration, all the work in literature only considers either of the optical techniques. In this paper, we aim to achieve improved image registration results by using the local-global optical techniques. The remaining part of this paper is as follows. Section 2 gives the details of local-global optical flow technique and the idea of apply it for image registration. Experiments are conducted in Section 3 to demonstrate the results and the quantitative registration errors are also given. Section 4 concludes this paper.

\section{METHODS}

In this section, we first discuss the original local and global methods, and further discuss how they can be combined and applied for image registration.

\subsection{Local-global optical flow}

According to experiments conducted, local method yields flow field which is more robust to noise and more accurate optical flow values. However, its operation time in MATLAB is much slower than global method. Another finding by the authors of this paper is that the larger number of iterations, the more accurate result will be. However, when the number of iterations reaches 500, the calculations of optic flow values will reach its limit.

Global method yields flow yields with $100 \%$ density, which means global method should fully represent the flow field, but are experimentally. Also, it is known to be more sensitive to noise. After we compile the global method program in MATLAB, the compilation time is less than one minute which is much faster than local method. However, Global method is not robust to noise.

Due to advantages and disadvantages of global and local methods, it is beneficial to combine these two methods to get a better one.

It is common to smooth the image values before calculating optic flow in order to get more clear results, and there are many existing smoothing techniques such as Gaussian filter using Gaussian calculations and Median filter which emphasize on the average values of each point. Combining the different smoothing effects can be done so that we can make use of the high robustness of local methods with full density of global techniques.

In an image domain $\Omega$ and time $t \quad[0, T] g(x, y, t)$ represents the image sequence within the image. Gaussian filter has been chosen to make use of its low-pass effect in order to remove noise and other destabilizing high frequencies. However, it is noted that too much pre-smoothing should be avoided else it will remove the original features of the image.

$$
f(x, y, t):=\left(K \sigma^{*} g\right)(x, y, t)
$$

where $\sigma$ represents standard deviation. Normal flow (weighting factor) is given by

$$
w_{n}=-\frac{f_{t}}{|\nabla f|} \frac{\nabla f}{|\nabla f|}
$$

Let us look at how we local method is represented in (Bruhn A and Weickert, 2005).

$$
\left.E_{L K}(u, v):=K_{p} *\left(\left(f_{x} u+f_{y} v+f_{t}\right)^{2}\right)\right)
$$

The standard deviation $\rho$ of the Gaussian serves as an integration scale over which the main 
contribution of the least square fit is computed. The larger the value of $\rho$, the more robust of the result will be under noise.

Global method is shown below in simplified form,

$$
E_{H S}(u, v)=\int_{\Omega}\left(\left(f_{x} u+f_{y} v+f_{t}\right)^{2}+\alpha\left(|\nabla u|^{2}+|\nabla v|^{2}\right)\right) d x d y(
$$

where $\Delta$ denotes the spatial Laplace operator $\Delta:=\partial_{x x}+\partial_{y y}$

So, we can easily derive the final combined localglobal method after some substitutions,

$$
E_{C L G 3}(\omega)=\int_{\Omega \times[0, T]}\left(\omega^{\mathrm{T}} J_{\rho}\left(\nabla_{3} f\right) \omega+\alpha\left|\nabla_{3} \omega\right|^{2}\right) d x d y d t
$$

and the weighted function has become

$$
\left|\nabla_{3} \omega\right|^{2}:=\left|\nabla_{3} u\right|^{2}+\left|\nabla_{3} v\right|^{2}
$$

\section{EXPERIMENTS AND RESULTS}

In order to determine the result of Combined LocalGlobal optic flow method, we have implemented it in MATLAB, so as local and global methods. In the following session, we will compare and discuss the effects of different methods.

First, we chose two groups of photos taken from subsequent time points in two different videos; the camera speed is around 25 frames/second.

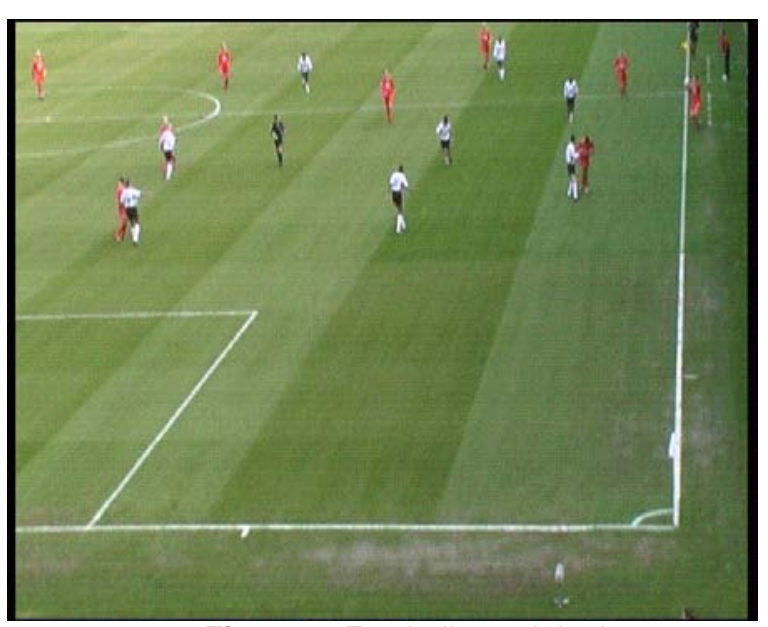

Figure 2: Football match in time t1

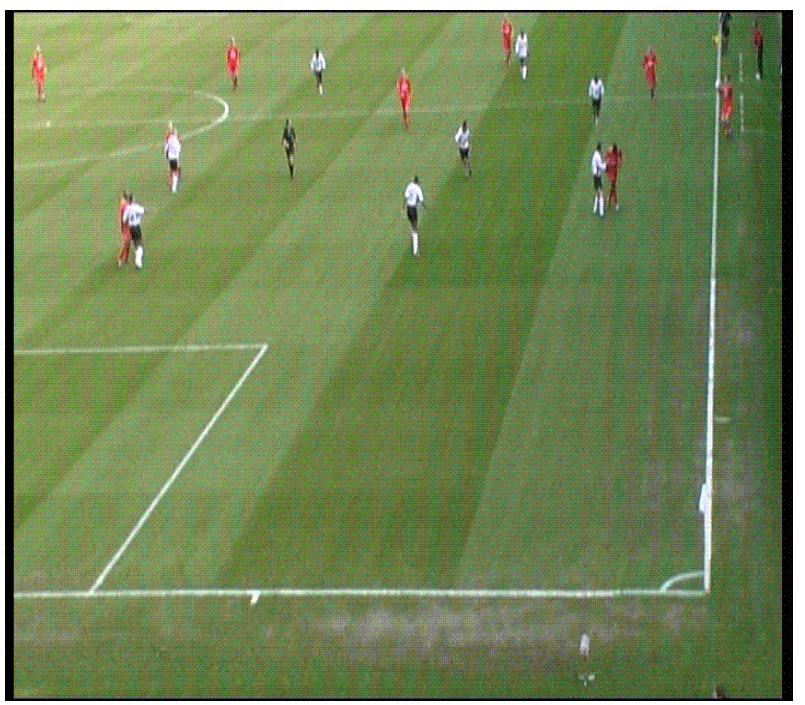

Figure 3:.Football match in time t2

Figures 2 and 3 are two photos taken with camera speed around 25 frames/sec. We used the two photos as inputs of global method, local method and combined local-global method in order to compare their results

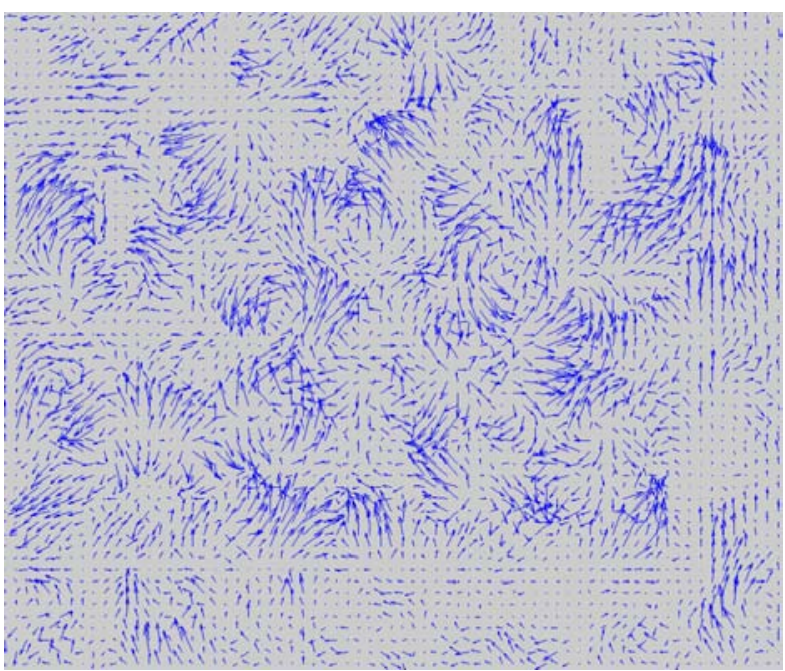

Figure 4: Global football match

In Figure 4, we can see that there is too much noise to affect the optic flow field, which means global method is not good enough to avoid the noises. 


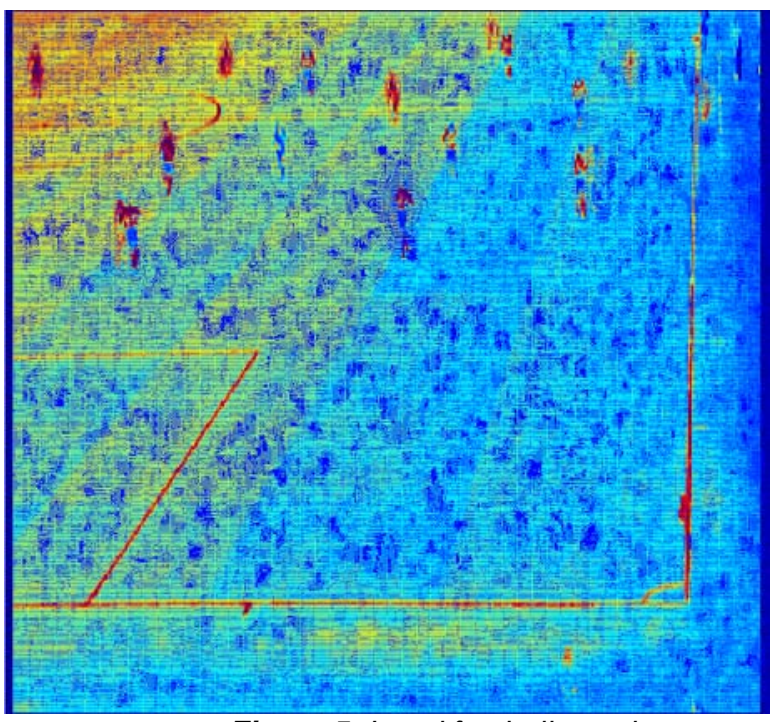

Figure 5: Local football match

In Figure 5, the overall view is much better than that in global method because we can see clear flow field under noise interferences.

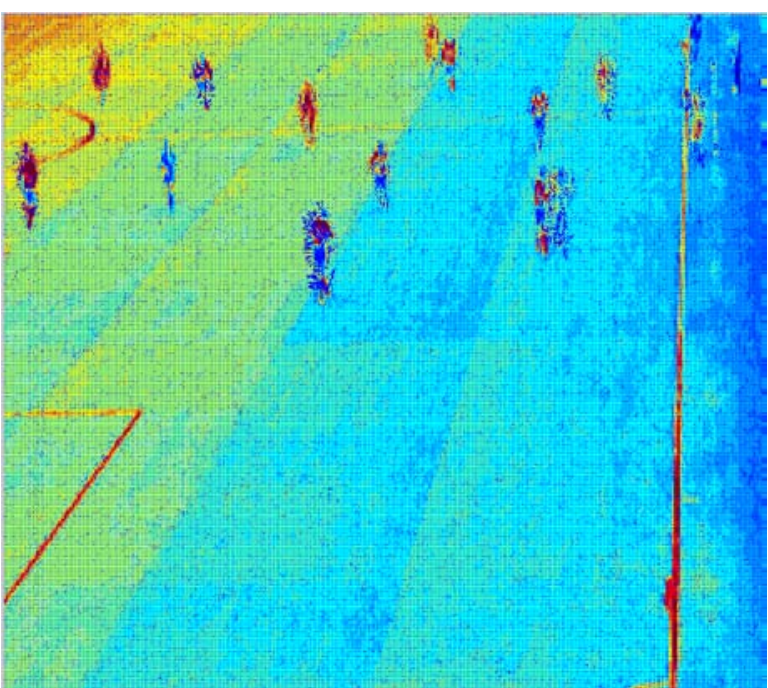

Figure 6: Combined local-global football match

From Figures 4,5 and 6 it is obvious that with the same inputs, the three different methods yield different output view. The three pictures are taken from the general view of the outputs, and it is clear that global method yields high density optic flow while local method yields more noise treatments. However, the CLG output show high contrast of optic flow density due to movement of objects to the stable objects and performs well under noise.

Generally speaking, from the overview pictures, optic flow in CLG output is more obvious around visible motion field while in local and global outputs; visible optic flow can be found throughout the whole pictures due to noise and other interferences. We chose the same part in Figures 4,5 and 6 and zoom into the flow fields. The results are shown in Figures 7, 8 and 9.

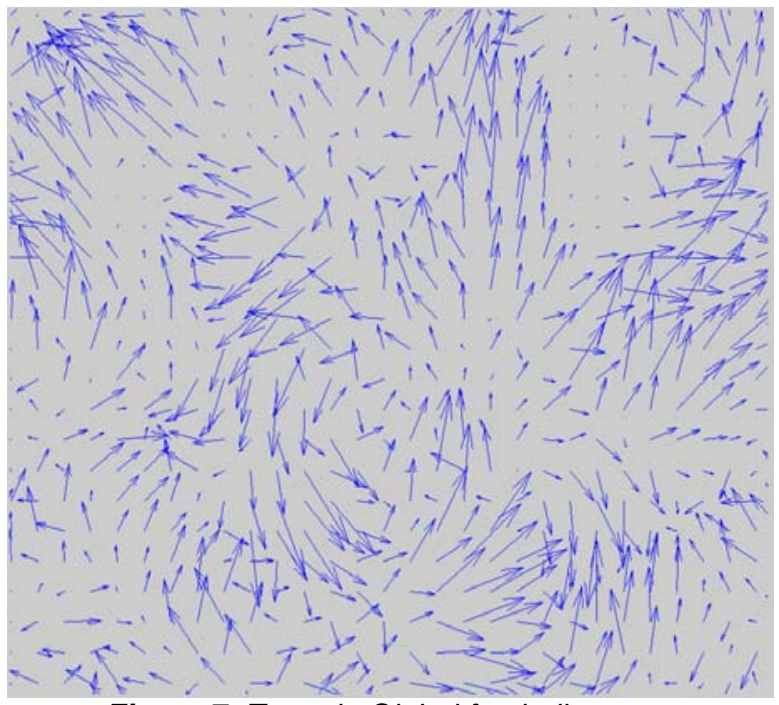

Figure 7: Zoom in Global football

In Figure 7 above, global flow fields are smooth and with high density.

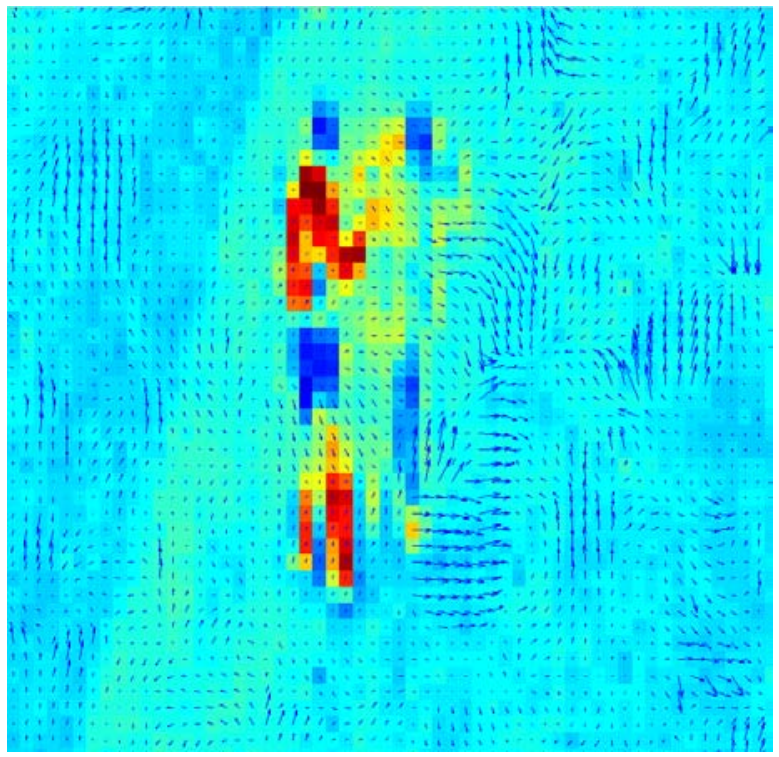

Figure 8: Zoom in Local football

In Figure 8, local optic flow filed is more robust under noise and with clear difference between moving and non-moving objects. 


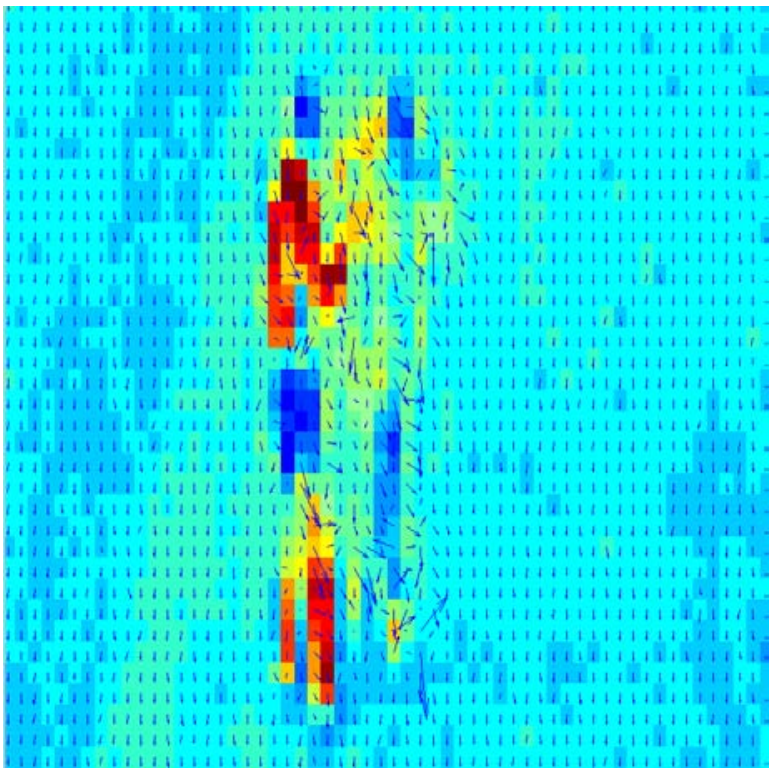

Figure 9: Zoom in CLG football

In Figure 9, we can see that optic flow field in CLG is very distinguishing between obvious and unobvious movements and robust to noise which have better view than global and local methods.

\section{CONCLUSION}

In this paper, the relation between image registration and optical flow estimation is discussed. The advantages and shortcomings of both local and global optical flow are introduced. The improved optical flow technique, namely LocalGlobal, which combines the advantages of both techniques, is applied for image registration. The experiments on images with standard image sequences and images with synthetic deformations show that this method is highly effective with the advantages of both local and global optical flow techniques. Our future work will be focused on refine this method and applied on more images such as in medical area.

\section{REFERENCES}

BARRON, J. L., FLEET, D. J. \& BEAUCHEMIN, S. S. 1994. Performance of optical flow techniques. Internation Journal of Computer Vision, 12, 43-77.

BRUHN A \& WEICKERT, J. 2005. Lucas/Kanade meets Horn/Schunck: Combining local and global optic flow methods. International Journal of Computer Vision, 61, 211-231.

COOPER, J. R. \& RITTER, N. J. Year. Optical flow for validating medical image registration. In: 9th IASTED International Conference on Signal and Image Processing, 2003. IASTED, 502-506.

LEFÉBURE, M. \& COHEN, L. D. 2001. Image registration, optical flow and local Rigidity. Journal of mathematical imaging and vision, 14, 131-147.

LIU, H., HONG, T.-H., HERMAN, M. \& CHELLAPPA, R. 1998. Accuracy vs efficiency trade-offs in optical flow algorithms. Computer Vision and Image Understanding, 72, 271-286.

PERIASWAMY, S. \& FARID, H. 2003. Elastic registration in the presence of intensity variations. IEEE Transactions on Medical Imaging, 22, 865874. 